BULLETIN OF THE

AMERICAN MATHEMATICAL SOCIETY

Volume 77, Number 5, September 1971

\title{
PERTURBATION OF PERIODIC MANIFOLDS OF HAMILTONIAN SYSTEMS
}

\author{
BY ALAN WEINSTEIN ${ }^{1}$ \\ Communicated by Emery Thomas, April 2, 1971
}

Introduction. It is well known that a closed orbit of a hamiltonian system is preserved under small perturbations of the hamiltonian function, provided that the orbit is nondegenerate in a certain sense $[5, \S 38]$. In this announcement, we describe some results about the behavior of manifolds of closed orbits of hamiltonian systems under perturbation of the hamiltonian function. This yields, in turn, new results concerning the existence of closed orbits near equilibria for which the linearized system exhibits degenerate behavior.

1. Periodic manifolds defined. Let $(M, \omega)$ be a symplectic manifold. (We follow the notation of [1], to which we refer the reader for definitions left unspecified here.) Any real-valued function $H$ on $M$ gives rise to a hamiltonian vector field $X_{H}$ on $M$. A submanifold $\Sigma \subseteq M$ is called a periodic manifold of $X_{H}$ if the following conditions are satisfied:

(PM 1). $\Sigma$ is an invariant manifold of $X_{H}$; i.e., $X_{H}$ is tangent to $\Sigma$ at all points of $\Sigma$.

(PM 2). All the orbits of $X_{H}$ on $\Sigma$ are closed, and their periods all divide a number $\tau>0$, called a period of $\Sigma$.

(PM 3). $H$ is constant on $\Sigma$.

Let $\left\{F_{t}\right\}_{t \in \mathbb{Q}}$ be the flow generated by $X_{H}$. If $\Sigma$ is a periodic manifold with period $\tau, F_{\tau}$ is the identity on $\Sigma$, and $T_{m} F_{\tau}$ maps $T_{m} M$ into itself for each $m \in \Sigma$. If $c$ is the value of $H$ on $\Sigma, T_{m} F_{r}$ maps $T_{m}\left[H^{-1}(c)\right]$ into itself and leaves the subspace $T_{m} \Sigma$ fixed. $T_{m} F_{\tau}$ induces, therefore, a map $T_{m}^{\nu} F_{\tau}$ of the "normal" space, $T_{m}\left[H^{-1}(c)\right] / T_{m} \Sigma$, into itself. We call $\Sigma$ nondegenerate if $T_{m}^{\nu} F_{\tau}$ does not have 1 as an eigenvalue for any $m \in \Sigma$.

For technical reasons connected with our method of proof, we need to impose a further condition on periodic submanifolds, called regularity. The precise definition is given in [11].

2. Examples. 1. If $\Sigma$ consists of a single closed orbit $\gamma, \Sigma$ is nondegenerate if and only if the characteristic multiplier 1 has multi-

AMS 1969 subject classifications. Primary 7034, 5750.

Key words and phrases. Symplectic manifold, hamiltonian system, periodic orbit, periodic manifold, small oscillations.

1 Partially supported by NSF Grant GP-20096. 
plicity 1 . A single closed orbit is always regular.

2. If $\Sigma$ is all of $H^{-1}(c)$, it is nondegenerate and regular.

3. What follows here is sometimes known as the "theory of small oscillations." (See [11] for a discussion of this terminology.)

Let $(M, \omega)$ be a symplectic vector space regarded as a symplectic manifold, $\omega$ being a constant section, and let $H$ be a quadratic hamiltonian. The vector field $X_{H}$, considered as a map from $M$ to itself, is linear, and the flow $\left\{F_{\tau}\right\}$ which it generates is given by

$$
F_{\tau}=\exp \left[\tau X_{H}\right]=\sum_{n=0}^{\infty} \frac{\tau^{n} X_{H}^{n}}{n !} .
$$

Let $V_{\tau} \subseteq M$ be the kernel of $F_{\tau}-I$, and suppose that $H$ is positivedefinite on $V_{\tau}$. (If $H$ is negative-definite on $V_{\tau}$, replace $H$ by $-H$.) Then $H^{-1}(1) \cap V_{\tau}$ is a compact, regular, periodic manifold for $X_{H}$, diffeomorphic to a sphere, which must be odd-dimensional because $X_{H}$ is a nowhere vanishing vector field on it. A short argument, using the definiteness of $H$ on $V_{\tau}$, shows that $H^{-1}(1) \cap V_{\tau}$ is nondegenerate. Finally, we remark that $V_{\tau}$ is the sum of the eigenspaces of $X_{H}$ corresponding to eigenvalues of the form $2 \pi n i / \tau$, where $n$ is any integer. The definiteness of $H$ on $V_{\tau}$ excludes the possibility that $n$ be zero. (If $H$ is nondegenerate but indefinite on $V_{\tau}$, one loses the compactness of $H^{-1}(1) \cap V_{\tau}$, which is essential for what follows.)

\section{The main theorem.}

THEOREM. Let $\Sigma \subseteq H^{-1}(c)$ be a compact, regular periodic manifold of $X_{H}$, with period $\tau$. Given any neighborhood $U_{1}$ of $\Sigma$ in $M$ and any number $\epsilon_{1}>0$, there exist

(a) a neighborhood $U$ of $\Sigma$ in $U_{1}$,

(b) a number $\epsilon, 0<\epsilon<\epsilon_{1}$,

(c) a neighborhood $\mathcal{G}$ of $H$ in the space $\mathfrak{F}(M)$ of real-valued functions on $M$ in the $C^{2}$-topology, and

(d) a mapping $P$ from $g$ to the space of 1-forms on $U$ in the $C^{1}$ topology, such that, for every $G \in \mathcal{G}$,

(i) the pullback $P^{c}(G)$ of $P(G)$ to $U \cap H^{-1}(c)$ is closed,

(ii) the zero set of $P^{c}(G)$ is equal to the set of closed orbits of $X_{G}$ on $U \cap H^{-1}(c)$ with periods dividing numbers in the interval $(\tau-\epsilon, \tau+\epsilon)$, and eters.

(iii) $P$ is continuous and preserves smooth dependence on param-

If $\Sigma$ is nondegenerate, then $\Sigma$ is a nondegenerate (in the sense of Bott [2]) zero manifold for $P^{c}(H)$. It follows that $U$ and $\mathcal{G}$ can be made small enough so that there exists 
(e) a mapping I from $\mathrm{g}$ to the space of embeddings of $\Sigma$ in $U$ in the $C^{1}$ topology, such that, for every $G \in \mathrm{g}$,

(iv) the image of $I(G)$ is contained in $G^{-1}(c)$,

(v) the zero set of the pullback of $P(G)$ to $I(G)$ is equal to the zero set of $P^{c}(G)$.

If $H^{1}(\Sigma, \Re)$ is zero, any closed 1 -form on $\Sigma$ is the differential of a real-valued function on $\Sigma$, which must attain a maximum and a minimum. A further topological argument implies that, if the dimension of $\Sigma$ is at least four, any function on $\Sigma$ whose critical point set is a disjoint union of circles must have at least three critical circles. The theory of functions whose critical point set is a union of circles is not very well developed, but I conjecture that every such function on a $(2 n-1)$-dimensional sphere must have at least $n$ critical circles. In any case, the theorem above has the following consequence:

CoRollary. Let $\Sigma \subseteq H^{-1}(c)$ be a compact, regular, nondegenerate periodic manifold of $X_{H}$, with period $\tau$. If $H^{1}(\Sigma, R)=0$, then, given any neighborhood $U$ of $\Sigma$ in $M$ and any $\epsilon>0$, there exists a $C^{2}$ neighborhood $\mathcal{Q}$ of $H$ in $\mathcal{F}(M)$ such that, for any $G \in \mathcal{G}, X_{G}$ has at least two closed orbits in $U$ with periods dividing numbers in $(\tau-\epsilon, \tau+\epsilon)$. If the dimension of $\Sigma$ is at least four, there are at least three such closed orbits.

4. Applications to the examples. 1. Since a closed form on the circle is not necessarily the differential of a function, our theorem as stated does not imply the stability of nondegenerate closed orbits mentioned in the Introduction. However, a version of the theorem for symplectic mappings can be combined with the existence of a global crosssection to derive the well-known result.

2. If $\Sigma$ is all of $H^{-1}(c)$, our results are related to those obtained by Moser [4], using the method of averaging. We eliminate Moser's requirement of nondegeneracy, and we need not restrict ourselves to perturbations depending on a real parameter. This generality is obtained at some expense, however-the closed 1 -forms obtained by the method of averaging are invariant under the action of $X_{H}$ on $\Sigma$, and this simplifies the topological problem of estimating the number of circles in their zero sets. In some sense, the method of averaging can be regarded as a first-order approximation to our method. It is suffcient in generic cases. More important to mention is that the reading of Moser's paper was an important stimulus for the present work.

3. If we wish to study motion in the neighborhood of an equilibrium point of a hamiltonian system, Darboux's theorem allows us to assume that $(M, \omega)$ is a symplectic vector space considered as a symplectic manifold. If $H$ is the given hamiltonian function, with a 
critical point at the origin, define $H_{\epsilon}$, for $0<\epsilon \leqq 1$, by $H_{\epsilon}(m)$ $=H(\epsilon m) / \epsilon^{2}$. By defining $H_{0}$ to be the hessian form of $H$ at the origin, we get a family $\left\{H_{\epsilon}\right\}$ of hamiltonian functions for which $H_{0}$ is quadratic and which is smooth as a function on $M \times[0,1]$. Furthermore, an easy computation shows that integral curves of $H_{\epsilon}$ are taken to integral curves of $H$ by the transformation $m \mapsto \epsilon m$. Now we can apply the results of $\S 3$. Let $i \lambda$ be a purely imaginary eigenvalue of $X_{H_{0}}$. Let $\tau=2 \pi / \lambda$. Then $V_{\tau}$ is the sum of the eigenspaces of $X_{H_{0}}$ corresponding to those eigenvalues which are integer multiples of $i \lambda$. If the restriction of $H_{0}$ to $V_{\tau}$ is positive-definite (and this is always the case when $H$ is the sum of kinetic and potential energy terms, where the potential energy has a nondegenerate critical point at the origin), then $H_{0}^{-1}(1) \cap V_{\tau}$ is a regular, nondegenerate periodic manifold for $X_{H_{0}}$, diffeomorphic to a sphere of dimension $2 n-1, n$ being the sum of the multiplicities of those eigenvalues which are positive integer multiples of $i \lambda$. If $n=1, H_{0}^{-1}(1) \cap V_{\tau}$ is a nondegenerate closed orbit, and we may conclude that, for $\epsilon$ near zero, $X_{H}$, has a nearby closed orbit on $H_{e}^{-1}(1)$, so that $X_{H}$ has a closed orbit on $H^{-1}\left(\epsilon^{2}\right)$, a result due to Liapunov. (See [1].) If $n$ is greater than 1 , then $S^{2 n-1}$ is simply connected, and the results of $\$ 3$ imply that, for $\epsilon$ near zero, $X_{H}$ has at least 2 closed orbits on $H^{-1}\left(\epsilon^{2}\right)$. If $n$ is greater than 2 , then $S^{2 n-1}$ has dimension greater than 4 , and $X_{H}$ has at least 3 closed orbits on $H^{-1}\left(\epsilon^{2}\right)$. If the conjecture in $\$ 3$ could be proven, it would imply that there are always at least $n$ closed orbits on $H^{-1}\left(\epsilon^{2}\right)$. Finally, let us note that our method seems to yield no results in case $H_{0}$ is indefinite on $V_{\tau}$, a case which occurs in physically interesting situations, including the restricted 3-body problem. The existence of closed orbits in such situations has been proven by Roels [7], using analytic methods akin to those originally used by Liapunov.

5. On the proof of the main theorem. A detailed proof of the results announced in this paper will appear in a subsequent publication [11]. The proof rests on two basic ideas. The first step is the usual one of using local transversal sections to reduce the search for closed orbits of a flow to the search for fixed points of a mapping. Since there is normally no global transversal section, one must use a family of local sections, as did Seifert in his study of certain vector fields on $S^{3},[6],[8]$. The second step involves the representation of symplectic mappings by closed 1-forms, as described by the author in [9] and [10]. A related technique was used by Meyer in a local setting [3].

\section{REFERENCES}

1. R. Abraham and J. Marsden, Foundations of mechanics, Benjamin, New York, 1967. MR 36 \#3527. 
2. R. Bott, Nondegenerate critical manifolds, Ann. of Math. (2) 60 (1954), 248-261. MR 16, 276.

3. K. R. Meyer, Generic bifurcation of periodic points, Trans. Amer. Math. Soc. 149 (1970), 95-107. MR 41 \#3931.

4. J. Moser, Regularization of Kepler's problem and the averaging method on a manifold, Comm. Pure Appl. Math. 23 (1970), 609-636.

5. H. Poincaré, Les mêthodes nouvelles de la mêcanique cêleste. Tome 1. Solutions périodiques. Non-existence des intégrales uniformes. Solutions asymptotiques, Paris, 1892; English transl., NASA TTF-450, National Aeronautics and Space Administration, Washington, D. C., 1967. MR 19, 414; MR 38 \#6135a.

6. G. Reeb, Sur un thêorème de Seifert sur les trajectoires fermées de certains champs de vecteurs, Internat. Sympos. Nonlinear Differential Equations and Nonlinear Mechanics, Academic Press, New York, 1963, pp. 16-21. MR 26 \#6502.

7. J. Roels, Extension aux cas résonants d'un thêorème de Liapunov sur les mouvements périodiques voisin d'un équilibre, Rapport $\mathrm{n}^{\circ}$. 33, Inst. Math. Pure Appl., Université Catholique de Louvain, 1969.

8. $\mathrm{H}$. Seifert, Closed integral curves in 3-space and isotopic two-dimensional deformations, Proc. Amer. Math. Soc. 1 (1950), 287-302. MR 12, 273.

9. A. Weinstein, Symplectic structures on Banach manifolds, Bull. Amer. Math. Soc. 75 (1969), 1040-1041. MR $39 \# 6364$.

10. - Symplectic manifolds and their Lagrangian submanifolds, Advances in Math. (to appear).

11. - Periodic orbits of hamiltonian systems via critical point theory, Preprint, Berkeley, Calif., 1971.

University of California, Berkeley, California 94720 